\title{
Variations in the leukocyte and cytokine profiles between placental and maternal circulation in pregnancy-associated malaria
}

This article was published in the following Dove Press journal: Research and Reports in Tropical Medicine

\author{
Okezie Caleb Okamgba' \\ Martin O Ifeanyichukwu' \\ Ayodele $O$ Ilesanmi $^{2}$ \\ Lawrence N Chigbu ${ }^{3}$ \\ 'Department of Medical Laboratory \\ Science, Faculty of Health Sciences \\ and Technology, Nnamdi Azikwe \\ University, Nnewi Campus, Nnewi, \\ Anambra State, ${ }^{2}$ Department of \\ Medical Laboratory Science, Kwara \\ State University, Malete, Kwara State, \\ ${ }^{3}$ Department of Microbiology, College \\ of Medicine, Abia State University, \\ Uturu, Abia State, Nigeria
}

Background: Activation of immune cells by malaria infection induces the secretion of cytokines and the synthesis of other inflammatory mediators. This study compared the cytokine levels and leukocyte count between malaria-infected peripheral and placental blood of pregnant women before delivery and postpartum. The cytokines assessed include interferon gamma (IFN- $\gamma$ ), tumor necrosis factor alpha (TNF- $\alpha$ ), interleukin-4 (IL-4), interleukin-6 (IL-6) and interleukin-10 (IL-10).

Materials and methods: The subjects comprised 144 malaria-infected pregnant women and 60 malaria-infected women at post-partum stage (for placental blood collection). Others were 60 malaria-uninfected pregnant women and 40 malaria-uninfected women at postpartum stage (for placental blood collection). Forty malaria-infected and 40 malaria-uninfected nonpregnant women served as control subjects. The test groups were asymptomatic, and the control groups were apparently healthy subjects. All were aged between 17 and 44 years. Ethical approval for the study was obtained at Abia State University Teaching Hospital and Living Word Mission Hospital, Aba. Informed consent was obtained from the participants. Blood samples were aseptically collected initially from the maternal peripheral circulation and from the placenta on delivery, and tested for HIV and malaria using standard methods. IFN- $\gamma$, TNF- $\alpha$, IL-4, IL-6 and IL-10 were measured by enzyme-linked immunosorbent assay technique. Kruskal-Wallis test was used for comparison of the groups.

Results: IFN- $\gamma$ was significantly higher in the peripheral than in placental blood $(P=0.001)$. IL-4 and IL-10 were significantly lower in the peripheral than in placental blood $(P=0.001$ and $P=0.004$, respectively). The total leukocytes, neutrophils and lymphocyte counts were significantly higher in the placenta than in peripheral blood $(P=0.001)$, and the mixed differential count was significantly higher in the placenta than in peripheral blood $(P=0.012)$.

Conclusion: This study has shown that the cytokine levels and leukocyte counts may differ between the peripheral and placental blood of the same women. Therefore, measurement of parameters in the peripheral circulation may not always reflect the levels in the placental blood for the assessment of immune cellular response at the materno-fetal interface.

Keywords: malaria parasite density, cytokines, peripheral blood, placental blood, leukocyte

\section{Introduction}

Malaria is a worldwide infection that affects about 500 million and kills more than 1 million people each year, with occurrence being majorly in the tropical and subtropical areas, especially in Africa, Asia, Central and South America. ${ }^{1}$ It is reported that malaria affects more than 3 million pregnant women each year in the developing countries, ${ }^{2}$ with a consequent toll on maternal and fetal mortality. ${ }^{3}$ The main effects
Correspondence: Ayodele O llesanm Department of Medical Laboratory Science, Kwara State University, I University Avenue, Malete 24I 104, Kwara State, Nigeria

Email ayodeleilesanmi2002@yahoo.com
Research and Reports in Tropical Medicine 2018:9 I-8

(c) (i) (5) 2018 0kamgba et al. This work is published and licensed by Dove Medical Press Limited. The full terms of this license are available at https://www.dovepress.com/terms. BY NC php and incorporate the Creative Commons Attribution - Non Commercial (unported, v3.0) License (http://creativecommons.org/licenses/by-nc/3.0/). By accessing the work permission for commercial use of this work, please see paragraphs 4.2 and 5 of our Terms (https://www.dovepress.com/terms php). 
of pregnancy-associated malaria include maternal anemia, low birth weight, preterm delivery and increased infant and maternal mortality. ${ }^{3}$ Some of these effects have been attributed to overexpression of cytokines and varied level of transmission. ${ }^{4,5}$ High cytokine level depresses cell-mediated immune response. Regarding the level of transmission in malaria-endemic areas, multiparous women and other adults acquire antibodies, thereby developing a considerable level of immunity that prevents them from presenting clinical malaria. However, the condition is different with primigravidae because they acquire antibodies late in pregnancy, resulting in low sustainable immunity. ${ }^{6}$

Infection of the peripheral blood begins when an infected female anopheles mosquito injects the sporozoite(s) into the subcutaneous tissue and less frequently, directly into the blood stream. The sporozoite(s) migrate to the liver, divide and mature successively to form thousands of merozoites. ${ }^{7}$ The invasion of the red blood cells (RBCs) by the merozoites results in clinical malaria, and the level of parasitemia may correlate with the severity of the disease. ${ }^{7,8}$

Parasitemia is influenced by cytoadherence or sequestration of the parasite in the internal organs. Sequestration is a phenomenon whereby malaria-infected erythrocytes accumulate in the microvasculature of various organs. The malarial parasite, Plasmodium falciparum, expresses on the surface of infected RBCs variant surface antigens, mainly the VAR2CSA molecule. ${ }^{9,10}$ The variant surface antigens mediate a range of interactions and adhere to receptors such as CD36 and ICAM1 on the host endothelial cells, platelets, uninfected erythrocytes and dendritic cells. The adherence to CD36 and ICAM1 results in acquiring antibody response. The principal VAR2CSA molecule is $P$. falciparum erythrocyte membrane protein 1 (PfEMP-1). ${ }^{5} \mathrm{P}$ EMP-1 is encoded by var genes and is expressed in the second half of the parasite life cycle. ${ }^{5}$ It is a virulent factor synthesized by the parasite during its maturation in host $\mathrm{RBCs}^{11}$ and is capable of attaching to host erythrocytes by making use of both host- and parasite-derived cytoskeletal adapters. ${ }^{12}$ The adhesion and interactions of the parasite/host adhesion molecules enhance the growth of the parasite in the internal organs. Sequestration in other organs may be mediated by receptors such as ICAM1 and CD36, but in the placenta, it is essentially mediated by chondroitin sulphate-A (CSA). ${ }^{6}$ In pregnancy, the PfEMP1 variant binds to the specific parasite ligand to CSA, and this development is responsible for pregnancy-associated placental malaria. ${ }^{10}$ CSA is a component of the proteoglycan matrix on placenta. It has been suggested that higher adherence to CSA results in greater binding and cooperativity. ${ }^{12}$ Determining the level of parasitemia is important while reporting malaria infection, since it is useful in the assessment of the parasite morphology, in clinical trials, epidemiologic studies and in the evaluation of effectiveness of interventions in individuals and communities. . $^{13,14}$

The placenta is the primary link between the mother and the fetus. It nourishes the fetus, eliminates fetal wastes and produces pregnancy hormones. ${ }^{15}$ Parasite-infected erythrocytes accumulate in the intervillous space, sometimes in higher density, and bind to CSA in the syncytiotrophoblast. ${ }^{12}$ Again, the placental intervillous spaces may be filled with macrophages, and these may contain hemozoin, antibodies and cytokines. Hemozoin probably remains for a long period until it is diluted out by the rapidly growing placenta. ${ }^{16}$

High malarial parasite infection is deleterious to the fetus because it induces some specific changes in the placenta. First, it triggers cellular immune response, which may result in the consumption of glucose and oxygen needed by the fetus. ${ }^{17}$ Second, it causes deposition of fibrinoid material and thickening of the cytotrophoblastic membrane. Sequestration of infected erythrocytes may lead to inflammatory response and poor placental function. It may also lead to structural and functional changes of the syncytiotrophoblast layer. ${ }^{18}$ These allude to a mechanical compromise of placental circulation, resulting in pathologic lesions and drastic reduction in the transport of nutrients to the fetus. ${ }^{18}$ Third, there is disruption of the normal immune balance, inducing inflammatory responses that result in increased synthesis of cytokines like tumor necrosis factor alpha (TNF- $\alpha$ ), interleukin (IL)-2 and interferon gamma (IFN- $\gamma$ ). ${ }^{13}$ An elevated level of TNF- $\alpha$ has been associated with effects that are inimical to placental survival and may result in low birth weight and anemia. ${ }^{5}$ Malaria in pregnancy is also capable of causing a disruption of trophoblast invasion, a phenomenon that may have profound effects on placental oxygenation and cause decreased placental volume and changes in uteroplacental and umbilical blood flow. ${ }^{18}$ On the other hand, increased IFN- $\gamma$ production may protect against prevalence of placental malaria, 5 and IL-10 may be beneficial in that it dampens proinflammatory responses induced by the sequestration phenomenon. ${ }^{19}$

Under normal conditions, changes in the cytokine profile occur in pregnant women and in transformation that takes place at the materno-fetal interface to ensure successful delivery of healthy infants. ${ }^{20}$ Moreover, there is a systematic transition to type- 2 cytokine dominance as pregnancy progresses to the third trimester, as overexpression of type-1 cytokines may compromise the viability of the fetus. ${ }^{16,21,22}$ The cytokines diffuse into the extracellular compartments 
and circulate in the peripheral blood. In response to invading pathogens, however, the cytokine profile is truncated and reversed to Th1 cytokine bias. One such condition that induces and reverses the cytokine bias to Th1 dominance is maternal malaria. Cytokines are agonists secreted principally by activated immune cells. They induce various transcription factors which, in turn, determine the fate of cells either for proliferation, differentiation, maturation or apoptosis.

Furthermore, leukocytes normally respond rapidly to combat infections and the polymorphonuclear cells appear to proffer the first line of defense. To enhance their functions, most cytokines, especially the Th17 group, known as powerful recruiters of immune cells, recruit more neutrophils, eosinophils and monocytes. While it is expected that in malaria, the total leukocyte count would be elevated, the wealth of evidence shows that it often appears low to normal in a phenomenon widely thought to reflect the localization of leukocytes away from circulation to the spleen and other marginal pools, but is not due to actual depletion or stasis. ${ }^{13,14}$ The extent to which leukocytes adhere to endothelial cells of internal organs, especially the placenta, during immune response could be assessed. Changes in the profile of both the maternal and placental cytokines and the blood immune cells should be expected in response to infections. However, the degree of variation induced by malaria parasitemia needs to be assessed. This study is, therefore, aimed at comparing the level of certain immunologic parameters between the peripheral and placental blood. These include the cytokine secretions and the blood cellular response to malarial infection in both compartments. This is with a view to assess the cellular immune response at the materno-fetal interphase which, to a large extent, also determines the viability of the fetus.

\section{Materials and methods}

This study, a follow-up research work that involved pregnant and postpartum women, was conducted in Aba, Abia State, Nigeria. Aba is a cosmopolitan town and the second largest commercial city of South Eastern Nigeria. It is located in latitude $05^{\circ} 10-\mathrm{N}$ and longitude $07^{\circ} 19-\mathrm{E}$, and at $205 \mathrm{~m}(673 \mathrm{ft})$ above the sea level. Aba is located $67 \mathrm{~km}$ from Umuahia, the state capital. ${ }^{23,24}$ The area experiences malaria transmission throughout the year. ${ }^{24,25}$ The stagnant water in the drainages provides adequate ecologic habitat for the breeding of mosquitoes. The mosquitoes are less susceptible to insecticides, possibly as a result of adaptation, and the majority of the study population sleep outside mosquito nets. ${ }^{24,25}$

The women recruited for this study were classified into six groups. The first and second groups were malaria parasit- emic and aparasitemic pregnant women. The third and fourth groups were subjects whose placental blood was infected and uninfected by malaria parasites, respectively. The fifth and sixth groups were malaria parasitemic and aparasitemic nonpregnant women, respectively. The malaria-uninfected pregnant women (group 2) and immediate postpartum women (group 4) were considered as the first batch of the test subjects, while the nonpregnant women (groups 5 and 6) were taken as the second batch of the control subjects. The test groups and control groups were asymptomatic and apparently healthy subjects, respectively. The classification into groups was based on detection of the malaria parasite in their blood and without the presentation of any form of clinical malaria. The participants were aged between 17 and 44 years, and all tested negative to human immunodeficiency virus (HIV). They had no history of liver diseases, hypertension, pre-eclampsia, malignancy, tuberculosis and gastric and duodenal ulcers. In addition, the pregnant groups had not complained of bleeding prior to 32 weeks of gestation, had not been transfused and were with singleton pregnancy. The criteria were applied to ensure that other sources of immune stimulation aside pregnancy and malaria were eliminated and to avoid any bias in interpretation. The immediate postpartum women had uncomplicated pregnancies and gave birth via vaginal route. On the contrast, subjects apparently known with chronic illnesses such as diabetes mellitus, cardiovascular diseases, liver diseases, tuberculosis and other infections, and inflammatory or pathologic disorders that may alter the immune response were excluded. Also excluded were those on hormonal drugs, HIV seropositives and those with multiple gestations. Those whose informed consent was not obtained were also excluded.

Ethical approval for the study was obtained from the Ethics Committees of two hospitals in Aba. They are Abia State University Teaching Hospital and Living Word Mission Hospital (Rhema University Teaching Hospital), both being tertiary hospitals with standard health facilities and manned by qualified and experienced health professionals. The pregnant and immediate postpartum subjects were recruited from the antenatal care units and delivery units, respectively, of the two hospitals, whereas the nonpregnant groups were students enrolled from the School of Nursing of Abia State University Teaching Hospital. Written informed consent of the individual subjects was obtained, and for subjects under 18 years of age, written informed consent of the parent or the husband, as the case may be, was obtained.

Peripheral sample was collected from the pregnant subjects at the last antenatal visit (few days to Expected Day of 
Delivery), while placental blood was collected from the same subjects shortly after delivery. Peripheral blood was collected by venipuncture: about $8 \mathrm{~mL}$ of blood was withdrawn from the antecubital vein of each subject by the phlebotomist. The blood from the placenta was obtained within 30 minutes of delivery and by biopsy pool method. Briefly, a block of tissue $(5 \times 5 \times 5 \mathrm{~cm})$ was excised from the cleaned maternal surface of the placenta, resulting in a large pool of intervillous blood at the excision site. About $8 \mathrm{~mL}$ of blood was quickly aspirated with a graduated sterile pipette. This was carried out by the hospital matrons.

Of the total blood collected, $3 \mathrm{~mL}$ was dispensed into EDTA anticoagulant bottle, mixed and set aside for the examination of malarial parasite, total leukocyte and differential leukocyte counts. The remaining $5 \mathrm{~mL}$ was allowed to clot in a pyrogen-free container and centrifuged at 3000 rpm for $10 \mathrm{~min}$. The serum thus obtained was used for the screening of HIV and estimation of cytokines: TNF- $\alpha$, IFN- $\gamma$, IL-4, IL-6 and IL-10.

Malaria parasitemia was determined by both rapid diagnostic testing and thick film methods. The malaria parasite density was evaluated using the quantitative parasite count (thick film by Giemsa staining technique). The HIV screening was done using Determine (Cat. No.: 240852/R7; Ref. No. 7D2343) and Immunocomb (Cat. No.: 10434503; Lot No.: 131015) test kits. The total leukocyte and differential leukocyte counts were evaluated using the automated three-parts hematology analyzer model KX21N (Sysmex Corporation, Kobe, Japan), while the cytokines were estimated by the enzyme-linked immunosorbent assay technique and the kits sourced from Abcam Company, Cambridge, UK (Lot Nos: IL-4 [ab100570], IL-6 [ab46027], IL-10 [ab46034], TNF [ab100654] and IFN [ab46025]). Procedure for each test was carried out according to the respective manufacturer's instructions.

\section{Statistical analysis}

All statistical analyses were performed using SPSS version 21. The results were expressed as mean and SD. KruskalWallis test was used for comparison of the groups. Level of significance was set at $P<0.05$.

\section{Results}

Table 1 shows a comparison of the mean $\mathrm{SD}$ of the cytokine levels and parasite density of all subjects, done using KruskalWallis test and post hoc analysis. It shows the effect of malaria infection and pregnancy on immune cell activation.

\section{Effect of malaria infection}

For this assessment, comparison was made between subjects of the groups 1 and 2, 3 and 4, 5 and 6, and 1 and 3. On comparing groups 1 and 2, values obtained for all the cytokines assessed were higher in malaria-infected pregnant group and were statistically significant when compared with the uninfected pregnant group.

When the values obtained for malaria-infected placental blood (group 3) were compared with those obtained for uninfected placental blood (group 4), there was increase in cytokine parameters in the former over the latter. However,

Table I Comparison of the mean \pm SD of cytokine and parasite density levels of malaria-infected and -uninfected subjects, done using Kruskal-Wallis test and post hoc analysis

\begin{tabular}{|c|c|c|c|c|c|c|}
\hline \multirow[t]{2}{*}{ Groups } & \multicolumn{5}{|c|}{ Cytokines (pg/mL) } & \multirow{2}{*}{$\begin{array}{l}\text { MP (parasite/ } \mu \mathrm{L}) \\
\text { MP }\end{array}$} \\
\hline & IFN- $\gamma$ & TNF- $\alpha$ & IL-4 & IL-6 & IL-I0 & \\
\hline GI $(n=144)$ & $22.94 \pm|2.7|$ & $21.12 \pm 12.57$ & $9.66 \pm 7.05$ & $32.11 \pm 27.92$ & $35.19 \pm 28.82$ & $658.56 \pm 484.55$ \\
\hline $\mathrm{G} 2(\mathrm{n}=62)$ & $5.98 \pm 3.11$ & $10.03 \pm 3.04$ & $7.17 \pm 3.91$ & $8.68 \pm 8.41$ & $14.76 \pm 6.17$ & - \\
\hline G3 $(n=60)$ & $11.71 \pm 6.55$ & $19.35 \pm 10.94$ & $14.86 \pm 6.37$ & $34.27 \pm 13.78$ & $55.57 \pm 43.13$ & $762.47 \pm 459.62$ \\
\hline G4 $(n=40)$ & $5.58 \pm 2.86$ & $|2.36 \pm 6.8|$ & $|2.03 \pm 5.0|$ & $26.99 \pm 12.65$ & $16.60 \pm 4.88$ & - \\
\hline G5 $(n=40)$ & $30.07 \pm 0.39$ & $13.17 \pm 0.33$ & $3.60 \pm 0.29$ & $23.42 \pm 0.45$ & $43.42 \pm 0.45$ & $837.75 \pm 737.53$ \\
\hline G6 $(n=40)$ & $4.69 \pm 2.64$ & $4.66 \pm 0.78$ & $2.13 \pm 0.36$ & $2.33 \pm 0.58$ & $6.45 \pm 4.15$ & - \\
\hline \multicolumn{7}{|l|}{ Post hoc } \\
\hline GI vs G2 & $0.001^{*}$ & $0.001^{*}$ & $0.040^{*}$ & $0.001^{*}$ & $0.001^{*}$ & \\
\hline GI vs G5 & $0.001^{*}$ & $0.001^{*}$ & $0.001^{*}$ & 0.502 & $0.006^{*}$ & 0.294 \\
\hline GI vs G3 & $0.001^{*}$ & 0.172 & $0.001^{*}$ & $0.068^{*}$ & $0.004^{*}$ & 0.213 \\
\hline G2 vs $\mathrm{G} 6$ & 0.111 & $0.001^{*}$ & $0.001^{*}$ & $0.001^{*}$ & $0.001^{*}$ & \\
\hline G3 vs G4 & $0.001^{*}$ & $0.005^{*}$ & 0.154 & 0.104 & $0.001^{*}$ & \\
\hline G5 vs G6 & $0.001^{*}$ & $0.001^{*}$ & $0.001^{*}$ & $0.00 I^{*}$ & $0.001^{*}$ & \\
\hline
\end{tabular}

Notes: The $\alpha$ level was set at $0.05, * P<0.05=$ significant, $P>0.05=$ not significant. GI, malaria-infected pregnant maternal peripheral blood; G2, malaria-uninfected pregnant maternal peripheral blood; G3, malaria-infected placental blood; G4, malaria-uninfected placental blood; G5, malaria-infected nonpregnant women's peripheral blood; G6, malaria-uninfected nonpregnant women's peripheral blood.

Abbreviation: MP, malaria parasite. 
the increased values for IL-4 and IL-6 were not statistically significant.

Comparison of values between malaria-infected nonpregnant women (group 5) and uninfected nonpregnant women (group 6) revealed significantly higher values for all the cytokine parameters assessed in the malaria-infected group than in the uninfected group.

Comparison of values between infected peripheral (group 1) and placental (group 3) compartments showed that IFN- $\gamma$ was significantly higher in infected peripheral than in infected placental blood, whereas IL-4 and IL-10 were significantly lower in infected peripheral than in placental blood. On the other hand, TNF- $\alpha$ and IL- 6 were not statistically significant.

\section{Effect of pregnancy}

For this assessment, comparison of values was made between groups 2 and 6 and between groups 1 and 5. On comparing malaria-uninfected pregnant women (group 2) vs malariauninfected nonpregnant women (group 6), IFN- $\gamma$ was mildly raised in the former, but there was no statistically significant difference. However, for the other cytokines, TNF- $\alpha$, IL-6, IL-4 and IL-10, the elevation in the values obtained for the uninfected pregnant women were statistically significant in all cases.

On comparing the values obtained from malaria-infected pregnant women (group 1) and those from infected, nonpregnant women (group 5), IFN- $\gamma$ and IL-10 were significantly lower in the former as compared with the latter, whereas TNF- $\alpha$ and IL-4 were significantly higher in the infected pregnant women than in infected nonpregnant women. IL-6 was not significantly expressed.

Table 2 shows a comparison of the mean \pm SD of the leukocyte counts between malaria-infected peripheral and placental blood, done using Kruskal-Wallis test. This table is for further assessment of immune cellular response between malaria-infected pregnant mothers' peripheral blood and malaria-infected placental blood (groups 1 vs 3).
The values obtained for total leukocytes, neutrophils, lymphocytes and mixed differential counts were higher in the infected placenta and statistically significant on comparison with the infected peripheral blood.

\section{Discussion}

Malaria in pregnancy is a common phenomenon in malariaendemic areas. It is an intractable disease that may affect the systemic circulation, placenta, brain and other internal organs. These are distinctive features of $P$. falciparum malaria, ${ }^{7}$ the species that is prevalent in the study environment.

This study shows that IFN- $\gamma$, TNF- $\alpha$, IL-4, IL-6 and IL-10 were elevated in malaria-infected pregnancies when compared to those in uninfected pregnant women. The result is similar to the findings of Szoba et $\mathrm{al}^{26}$ and Nmorsi et al, ${ }^{27}$ respectively, who reported that IFN- $\gamma$ and IL- 6 were significantly elevated in malaria-infected pregnant women than in uninfected nonpregnant women, and to that of Torre et al, ${ }^{28}$ who reported significantly elevated levels of IFN- $\gamma$, TNF- $\alpha$ and IL-10 in malaria-infected pregnant women than in their control counterparts. The same is also true when compared with Bayoumi et $\mathrm{al}^{29}$ and Boström et $\mathrm{al},{ }^{30}$ respectively, who reported significantly increased IL-10 in malaria-infected pregnant women than in uninfected control pregnant women. On the other hand, the discordance with the results of Bayoumi et $\mathrm{al}^{29}$ and Boström et al, ${ }^{30}$ respectively, which showed that IFN- $\gamma$, IL-4 and IL-10 were elevated in uninfected than in malaria-infected peripheral blood, could be due to the regional differences, since our study population is a high malaria density zone. In the studies of Megnekou et $\mathrm{al}^{19}$ and Wilson et al, ${ }^{31}$ IL-10 was raised significantly in malariapositive pregnant women than in the ones who had no malaria.

Another striking finding of this study was that the levels of IFN- $\gamma$, TNF- $\alpha$ and IL-10 were increased in malaria-infected placenta than in malaria-uninfected placenta. This result is similar to the finding of Suguitan et al. ${ }^{32}$ The significantly increased expression of IL-10 was due to the fact that this regulatory cytokine characterizes normal human pregnancy.

Table 2 Comparison of mean $\pm S D$ of the leukocyte count between infected peripheral and placental blood (using Kruskal-Wallis test)

\begin{tabular}{llll}
\hline Parameters & Peripheral blood $(\mathbf{n}=\mathbf{1 4 4})$ & Placental blood $(\mathbf{n}=60)$ & $P$-value \\
\hline Total leukocytes $\left(\times 10^{9} / \mathrm{L}\right)$ & $7.09 \pm 2.02^{\mathrm{a}}$ & $11.00 \pm 4.80^{\mathrm{b}}$ & 0.001 \\
Neutrophils $\left(\times 10^{9} / \mathrm{L}\right)$ & $3.9 \pm 0.91^{\mathrm{a}}$ & $5.0 \pm 0.78^{\mathrm{b}}$ & 0.001 \\
Lymphocytes $\left(\times 10^{9} / \mathrm{L}\right)$ & $2.3 \pm 0.77^{\mathrm{a}}$ & $4.9 \pm 0.89^{\mathrm{b}}$ & 0.001 \\
MXD leukocyte $\left(\times 10^{9} / \mathrm{L}\right)$ & $0.9 \pm 0.41^{\mathrm{a}}$ & $1.1 \pm 0.25^{\mathrm{b}}$ & 0.012 \\
\hline
\end{tabular}

Notes: This table shows a comparison of the mean \pm SD of the leukocyte counts between malaria-infected peripheral and placental blood, done using Kruskal-Wallis test. It is for further assessment of immune cellular response between malaria-infected pregnant mothers' peripheral blood and malaria-infected placental blood (groups I vs 3 ). The differences in values between the two compartments were all significant. The $\alpha$ level was set at 0.05 . a,b Show statistical difference. Values not sharing the same superscript mean there is a statistical difference. Values sharing the same superscript mean there is no statistical difference.

Abbreviation: MXD, mixed differential leukocytes comprising monocytes, eosinophils and basophils. 
In placental malaria, IL-10 concentrations are elevated, ${ }^{32}$ in order to suppress the effects of increased levels of Th1 cytokines. IL-10 has a major role in controlling inflammatory responses and preventing damage of materno-fetal placental barrier. ${ }^{33,34}$ Megnekou et al ${ }^{19}$ found that IL-10 increases are associated with infection by $P$. falciparum during pregnancy, suggesting that IL-10 could be used as a biomarker of falciparum malaria in pregnancy. As a consequence to the high parasite density found in placenta in our study, it may be concluded that comparatively higher IFN- $\gamma$, TNF- $\alpha$ and IL-10 levels should be expected in this compartment.

On comparing the concentrations of cytokines in the infected systemic and local placental blood, the finding of this study showed that IFN- $\gamma$, a proinflammatory cytokine, was reduced in the placenta than in peripheral blood and IL-4 and IL-10, the anti-inflammatory cytokines, were increased in the placenta than in peripheral blood. This finding is in agreement with earlier reports by Wegmann et $\mathrm{al}^{33}$ and Sacks et al, ${ }^{20}$ respectively, which posited that anti-inflammatory cytokines were more expressed in the placental environment than the proinflammatory cytokines. This phenomenon is protective to the placenta and the fetus against inflammation, malaria parasite invasion and rapid metabolism induced by growth factors for fetal development. Moreover, the placenta can influence the systemic cytokine profile, depending on the cytokine of interest.

Overall, the finding presented herein agrees with earlier studies showing that malaria induces immune stimulation ${ }^{35}$ which is accompanied by the secretion of various cytokines. According to D'Ombrain et al, ${ }^{36}$ phagocytosis of the parasite, the hemozoins or glycosylphosphatidylinositol and the parasite toxin by the phagocytic cells causes immune stimulation, resulting in the secretion of cytokines. ${ }^{36}$ Moreover, reactive oxygen species such as hydrogen peroxide $\left(\mathrm{H}_{2} \mathrm{O}_{2}\right)$, hydroxyl $\left(\mathrm{OH}^{-}\right)$and superoxide $\left(\mathrm{O}_{2}^{-}\right)$generated during oxidative stress activate the leukocytes with the release of more cytokines. ${ }^{37}$ Another factor that contributes to the secretion of cytokines during malaria is the initiation of the coagulation cascade consequent to endothelial damage. The endothelial walls could be injured or damaged due to sequestration and adherence of the parasite. It is pertinent to note that with damage of the endothelial walls, hemostatic activities are initiated for the repair of injured vessels and for preventing blood loss. Most plasma proteins, especially the coagulation factors that are initiated in the process, are inflammatory mediators, and the release of an inflammatory mediator, such as coagulation factors and cytokines, causes the initiation or activation and the concomitant secretion and release of another inflammatory mediator. ${ }^{37}$ These results imply that the levels of cytokines are raised by malaria infection.
Furthermore, when the values of the cytokines were compared between the malaria-uninfected pregnant women's peripheral blood (group 2) and malaria-uninfected nonpregnant women's peripheral blood (group 6), there was significant elevation of TNF- $\alpha$, IL-4, IL-6 and IL-10 in the former, but with IFN- $\gamma$, there was no significant difference. Data obtained in this study are in accordance with this notion and are supported by those obtained by Sacks et al, ${ }^{20}$ in which the cytokine levels were higher in pregnant compared to those in nonpregnant subjects. In cyesis, the cytokines in the plasma or serum are initially predominantly type- 1 because the circulating monocytes are primed to produce Th1 cytokines, ${ }^{30}$ which are crucial in immune surveillance against pathogens. ${ }^{20}$ However, as pregnancy progresses, there is systemic transition to type- 2 cytokines because overexpression of type-1 cytokines is deleterious to the viability of the fetus. ${ }^{16}$ This finding reaffirms that normal pregnancy is considered to be a state of controlled maternal mild inflammation, where the levels of proinflammatory and regulatory cytokines are raised compared to those in nonpregnant states.

In addition, the data in this study revealed that the total leukocyte count was increased in the infected placenta than in peripheral blood. This is in agreement with the aforementioned reports that the uterus, endometrium, placenta and trophoblasts are surrounded by monocytes, T cells and natural killer cells. ${ }^{38}$ These immune cells may be positioned to create an enabling environment such as the regulation of the cytokine milieu, in order to ensure survival of the fetus by warding off possible invasion of the placenta by pathogens. It also suggests that a good number of white blood cells would be recruited and these adhere to the vascular endothelial cells during inflammatory or immune response, in this case, for protection against the parasitic invasion of the placenta. In addition, the level of leukocyte recruitment may depend on the parasite density. Increased parasite density may attract an increased and corresponding number of leukocytes.

Data obtained on the differential white blood cell count show the number and type of leukocyte cell line that may be preferentially increased against the parasite pathogen in the peripheral and placental blood. In response to stress, such as inflammation or infection, the polymorphonuclear neutrophils in the storage pool are released into the circulation. Normally, neutrophils are released in large numbers predominantly more than any other leukocyte series. Chemotaxis is greatly pronounced with neutrophils than with any other leukocyte type, and this is expected to happen in order that appropriate cellular response may match the increased parasite density in the placenta. This could account for the 
elevated levels of the neutrophils in response to placental malaria infection.

Regarding the lymphocyte series, this study found that the lymphocytes were elevated in the placenta than in peripheral blood in malaria-infected subjects. On immune activation, the thymus-dependent lymphocytes ( $T$ cells) divide into two populations - the effector lymphocytes which live for 2 days and the memory lymphocytes that reverse to resting stage and survive for many years. ${ }^{39}$ The disappearance of the memory lymphocytes from the circulation to the tissues such as the placenta could create an imbalance, favoring the number of lymphocytes in the placenta than in peripheral blood.

In the mixed differential category which comprises monocytes, eosinophils and basophils, these cells are known to move freely to engulf and digest microbes. Aside phagocytosis, the monocytes specifically play the all-important role as antigen-presenting cells in the peripheral blood, thereby enhancing the innate immune response. ${ }^{39}$ Under normal conditions, monocytes/macrophages are positioned in the uterus, endothelium, placenta and trophoblasts, and given the fact that the number of monocytes released by hemopoietic progenitor cells greatly outnumbers the eosinophils and basophils, it is expected that the number of mixed differential white cells would have been higher in the placenta than in peripheral blood. However, granted that there is massive release of monocytes to the tissues, it is likely that the rate of attrition of these cells is higher in the placenta because of the inflammatory processes cited earlier.

\section{Conclusion}

This study has shown that malaria of pregnancy has the propensity to induce immune activation with the release of cytokines; however, variations exist in the measurement of parameters between the placenta and the peripheral blood. IFN- $\gamma$ is reduced in infected placenta than in peripheral blood, whereas IL-10 and IL-4 are elevated (possibly to suppress the expression of IFN- $\gamma$ ) in infected placenta than in systemic circulation. In addition, the leukocyte counts may be raised in the placenta than in peripheral blood. Therefore, measurement of parameters in the peripheral circulation may not be reflective of the cellular immune response in the placental compartment of the same women. Consequently, administration of interventions on the assumption that the measurements obtained from the peripheral blood would mimic activities at the local materno-fetal interface could be misleading and may not achieve the desired results. Moreover, it is suggested that clinicians commence interventions early enough during antenatal care, especially in malaria-endemic areas, in order to promptly check the effect of malaria infection.

\section{Acknowledgments}

We are grateful to the pregnant women at the antenatal clinics and the immediate postpartum women who voluntarily participated in this study. We thank Professor IM Ekejindu and others in the Department of Medical Laboratory Science, Nnamdi Azikiwe University, who made useful inputs in the design of this study. Dr Nkiru Nwagbo, Clara Igwe and JI Iruka are acknowledged for their assistance in collecting placental specimens. We are also indebted to the following institutions where the measurements were carried out: parasitology and hematology indices were carried out at Abia State University Teaching Hospital, Aba; enzymelinked immunosorbent assay and other serologic investigations were performed at the Research Units of Living Word Mission Hospital and New Covenant Laboratories Ltd, Aba. Finally, we wish to thank Victor Akidi and Chinelo Okezie for their efforts in carrying out the statistical analysis of this study.

Abcam Company, UK, and Monobind Incorporated, USA, were kind enough to provide the cytokines and biochemical kits, respectively.

This study was supported by a grant from B.M.S Links.

\section{Disclosure}

The authors report no conflicts of interest in this work.

\section{References}

1. Warren L. Cellular basis of the immune response. Review of Medical Microbiology and Immunology. 11 th ed. USA: McGraw Hill Company Inc.; 2010:384-401.

2. Abrams ET, Kwiek JJ, Mwapasa V, et al. Malaria during pregnancy and foetal hematological status in Blantyre, Malawi. Malaria J. 2005;4(1):39-46.

3. Falade CO, Tongo OO, Ogunkunle OO, Orimadegun AO. Effects of malaria-in-pregnancy on newborn anthropometry. J Infect Dev Ctries. 2010;4(7):448-453.

4. Takem EN, D'Alessandro U. Malaria in pregnancy. Mediterr J Hematol Infect Dis. 2013;5(1):e2013010.

5. Rogerson SJ, Mwapasa V, Meshnick SR. Malaria in pregnancy: linking immunity and pathogenesis to prevention. Am JTropical Med Hygiene. 2007;77(6).

6. Iona OD, Rajeshwara NA, Sean TA, et al. Gravidity-dependent production of antibodies that inhibit binding of plasmodium falciparuminfected erythrocytes to placental chondroitin sulfate proteoglycan during pregnancy. Infect Immun. 2001;69(12):7487-7492.

7. Arora DR, Arora BB. Malaria parasite In: Medical Parasitology. 3rd ed. New Delhi, India: CBS Publishers distribution Ltd; 2010: 71-86.

8. Phillips A, Bassett P, Zeki S, Newman S, Pasvol G. Risk factors for severe disease in adults with falciparum malaria. Clin Infect Dis. 2009;48(7):871-878.

9. Tangpukdee N, Krudsood S, Kano S, Wilairatana P. Falciparum malaria parasitaemia index for predicting severe malaria. Int $J$ Lab Hematol. 2012;34(3):320-327.

10. Doritchamou J, Bertin G, Moussiliou A, et al. First-trimester Plasmodium falciparum infections display a typical "placental" phenotype. J Infect Dis. 2012;206(12):1911-1919.

11. Smith JD, Miller LH. Infected erythrocytes bind to hyaluronic acid and malaria in pregnant women. J Infect Dis. 2004;189(2):165-168. 
12. Chishti AH. Malaria selectively targets pregnancy receptors. Blood. 2015;125:217-218.

13. Ellis MF, Wendy AP, Allan JM, et al. White blood cell counts and malaria. J Infect Dis. 2005;192(2):323-330.

14. Ifeanyichukwu MO, Esan AJ. Evaluation of blood cells and platelets in Plasmodium falciparum malaria infected individuals. Int J Hematol Dis. 2014;1(1):49-54.

15. Ashwood ER, Knight GJ. Clinical chemistry of pregnancy. In: Burtis CA, Ashwood Er, Burns DE, editors. Tietz Textbook of Clinical Chemistry and Molecular Diagnostics. 4th ed. St. Louis, MO: Elsevier Saunders; 2006:2153-2206.

16. Maestre A, Carmona-Fonseca J. Immune responses during gestational malaria: a review of the current knowledge and future trend of research. J Infect Dev Ctries. 2014;8(4):391-402.

17. Uneke CJ. Impact of placental Plasmodium falciparum malaria on pregnancy and perinatal outcome in Sub-Saharan Africa II: effects of placental malaria on perinatal outcome, malaria and HIV. Yale J Biol Med. 2007;80(3):95-103.

18. Schmiegelow C, Minja D, Oesterholt M, Pehrson C, Suhrs HE, Boström S. Malaria and fetal growth alterations in the 3rd trimester of pregnancy: a longitudinal ultrasound study. PLoS One. 2013;8(1):e53794.

19. Megnekou R, Lissom A, Bigoga JD, Djontu JC. Effects of pregnancy associated malaria on $\mathrm{T}$ cell cytokines in Cameroonian women. Scand J Immunol. 2015;81(6):508-514.

20. Sacks GP, Studena K, Sargent K, Redman CW. Normal pregnancy and pre-eclampsia both produce inflammatory changes in peripheral blood leukocytes akin to those of sepsis. Am J Obstet Gynecol. 1998;179(1): $80-86$.

21. Halonen M, Lohman IC, Stern DA, et al. Th1/Th2 patterns and balance in cytokine production in the parents and infants of a large birth cohort. J Immunol. 2009;182:3285-3293.

22. Denney JM, Nelson EL, Wadhwa PD, et al. Longitudinal modulation of immune system cytokine profile during pregnancy. Cytokines. 2011;53(2):170-177.

23. National Population Commission (2006). The Nigeria 2006 Census Figure. Available from: http://population.gov.ng/publications/. Accessed November 20, 2017.

24. Kalu MK, Obasi AN, Nduka FO, Christian GO. A comparative study of the prevalence of malaria in Aba and Umuahia Urban Areas of Abia State, Nigeria. Res J Parasitol. 2012;7(1):17-24.

25. Ezeigbo OR, Osuagwu MC, Ezuike MN, Ibegbulam ZO, Kalu S. Malaria Parasitaemia in children aged 1-5 years in Aba, South Eastern Nigeria. Int J infect Dis. 2014;21(Suppl 1):165.
26. Szoba SJ, Sullivan BM, Peng SI, Glimcher LH. Molecular mechanisms regulating $\mathrm{T}_{\mathrm{H}} 1$ immune responses. Ann Rev Immunol. 2003;21:713-758.

27. Nmorsi OPG, Isaac C, Ohaneme BA, Obiazi HAK. Pro-inflammatory cytokine profiles in Nigerian pregnant women infected with Plasmodium falciparum malaria. Asian Pac J Trop Med. 2010;3(9):731-733.

28. Torre D, Speranza F, Giola M, Matteelli A, Tambini R, Gilberto B. Role of $\mathrm{T}_{\mathrm{H}} 1$ and $\mathrm{T}_{\mathrm{H}} 2$ cytokines in immune response to uncomplicated malaria. Clin Diagn Lab Immunol. 2002;9(2):348-351.

29. Bayoumi NK, Bakhet HK, Mohammed AA, et al. Cytokine profiles in peripheral, placental and cord blood in an area of unstable malaria transmission in Eastern Sudan. J Trop Pediatr. 2008;54(4):202-204.

30. Boström S, Ibitokou S, Oesterholt M, et al. Biomarkers of Plasmodium falciparum infection during pregnancy in women living in Northeastern Tanzania. PLoS One. 2012;7(11):e48763.

31. Wilson NO, Bythwood T, Solomon W, et al. Elevated levels of IL-10 and G-CSF associated with asymptomatic malaria in pregnant women. Infect Dis Obstet Gynecol. 2010;2010.

32. Suguitan AL, Cadigan TJ, Nguyen TA, et al. Cytokine changes in the placenta of women with pre-term deliveries in Yaounde, Cameroon. $\mathrm{Am}$ J Trop Med Hyg. 2003;69(6):574-581.

33. Wegmann TG, Lin H, Guilbert L, Mosman TR. Bidirectional cytokine interactions in the maternal-foetal relationship: is successful pregnancy a $\mathrm{T}_{\mathrm{H}} 2$ phenomenon? Immunol Today. 1993;14(7):353-356.

34. Bouyou-Akotet MK, Kombila M, Kremsner PG, Marrungou E. Cytokine profiles in peripheral, placental and cord blood in pregnant women from an area endemic for Plasmodium falciparum. Eur Cytokine Netw. 2004;15(2):120-125.

35. Rowe JA, Kyes SA. The role of Plasmodium falciparum var genes in malaria in pregnancy. Mol Microbiol. 2004;53(4):1011-1019.

36. D'Ombrain MC, Robinson LJ, Stanisic DI, et al. Association of early interferon-gamma production with immunity to clinical malaria: a longitudinal study among Papua New Guinean children. Clin Infect Dis. 2008;47(11):1380-1387.

37. Kumar V, Abbas AK, Fausto N, Aster JC. Acute and chronic Inflammation. In: Robbins and Cotran. Pathological Basis of Diseases. 8th ed. Philadelphia: Elsevier Saunders; 2010:43-78.

38. Germain SJ, Sacks GP, Soorana SR, Sargent IL, Redman CW. Systemic inflammatory priming in normal pregnancy and preeclampsia: the role of circulating syncytiotrophoblast microparticles. J Immunol. 2007;178(9):5949-5956.

39. Venugopal J. Cytokines. In: Fundamentals of Medical Immunology. 1st ed. New Delhi, India: Jaypee Brothers Medical Publishers Ltd., 2007:123-135.
Research and Reports in Tropical Medicine

\section{Publish your work in this journal}

Research and Reports in Tropical Medicine is an international, peerreviewed, open access journal publishing original research, case reports, editorials, reviews and commentaries on all areas of tropical medicine, including: Diseases and medicine in tropical regions; Entomology; Epidemiology; Health economics issues; Infectious disease; Laboratory

\section{Dovepress}

science and new technology in tropical medicine; Parasitology; Public health medicine/health care policy in tropical regions; and Microbiology. The manuscript management system is completely online and includes a very quick and fair peer-review system. Visit http://www.dovepress. com/testimonials.php to read real quotes from published authors. 